\title{
Uso de reducción de daños y gravedad psicopatológica y médica en pacientes en programa de mantenimiento con metadona
}

\author{
Harm reduction program use, psychopathology and medical \\ severity in patients with methadone maintenance treatment
}

\author{
Nieves Martínez-Luna***,***, Laia Rodriguez-Cintas***, Abderraman Esojo*,**, Raúl Felipe \\ Palma-Álvarez***, María Robles-Martínez*, **, Lara Grau-López*******, Marta Perea***, \\ CARlos Roncero*,**,***,****.
}

*Sección de Adicciones y Patología Dual. Servicio de Psiquiatría Hospital Universitario Vall d'Hebrón. ASPB, Barcelona (España). **Servicio de Psiquiatría. Hospital Universitario Vall d'Hebrón. Barcelona- CIBERSAM (España).

***Departamento de Psiquiatría. Universidad Autónoma de Barcelona. (España).

**** Psychiatric Service. University of Salamanca Health Care Complex (Complejo Asistencial Universitario de Salamanca/ CAUSA), Salamanca, Spain. Institute of Biomedicine of Salamanca (IBSAL). University of Salamanca (Spain).

\section{Resumen}

Los programas de mantenimiento con metadona (PMM) para el tratamiento de la dependencia a opiáceos han demostrado elevada eficacia, siendo los más utilizados en la actualidad. La Reducción de Daños (REDAN) se refiere a tratamientos y abordajes complementarios en personas con graves dificultades para la abstinencia y conductas de riesgo. El objetivo del presente trabajo es comparar pacientes en PMM que utilizan servicios REDAN (PMM-REDAN) versus pacientes en PMM que no utilizan servicios REDAN (PMM-NO REDAN). Se incluyó un total de 143 pacientes en PMM (PMM-REDAN = 42, PMM-NO REDAN = 101) y se realizó un subanálisis de los menores de 45 años ( $\mathrm{n}=116$; 38 PMM-REDAN, 78 PMM-NO REDAN). Se hizo una evaluación de datos socio-demográficos, EuropASI, SCID-I y SCID-II. Los pacientes PMM-REDAN son más jóvenes, utilizan la vía parenteral y presentan trastornos de personalidad Clúster B comórbidos a la adicción. Los PMM-NO REDAN consumen más por vía fumada y tienen dosis bajas de metadona. Los menores de 45 años PMM-REDAN son más jóvenes, tienen mayor prevalencia de enfermedades hepáticas, utilizan más la vía parenteral, tienen un consumo de drogas más grave y menos soporte socio-familiar en las subescalas EUROP-ASI que los menores de 45 años PMM-NO REDAN. En conclusión, los pacientes PMM-REDAN son más jóvenes, reciben dosis mayores de metadona y utilizan vías de mayor riesgo, hecho que implica un inicio temprano en prácticas de consumo de riesgo, mayor tiempo de exposición a la heroína, con consecuencias de mayor gravedad de la adicción, mayor comorbilidad infecciosa, médica y psiquiátrica, siendo un grupo vulnerable y con pronóstico desfavorable. Palabras clave: programa de reducción de daño; tratamiento de mantenimiento con metadona; VIH; hepatitis C; dependencia heroína.

\begin{abstract}
Methadone maintenance programs (MMP) for opioid dependence treatment have been widely used due to their effective therapeutic outcomes. Harm reduction programs (HRP) are complementary programs for severe patients with high risk behaviors and when abstinence is not possible. This study aims to compare patients in MMP that use HRP (MMP-HRP) and patients in MMP who do not use HRP (MMP-NO HRP). The sample was composed of 143 patients (MMP-HRP $=42$ vs. MMP-NO HRP $=101$ ). An additional subanalysis was performed with patients under 45 years of age $(n=116$; MMP-HRP $=38$ vs. MMP-NO HRP $=78)$. All patients were assessed with an ad hoc socio-demographic questionnaire, EuropASI, SCID-I, and SCID-II. Results show that MMP-HRP patients were younger with more frequent use of intravenous drugs and with a high prevalence of Cluster B personality disorders. MMP-NO HRP patients had lower methadone doses compared to MMP-HRP patients and preferred to use drugs by smoked route more frequently. In the subanalysis of patients under 45, MMP-HRP patients were younger, had a higher prevalence of liver diseases, more intravenous drug use, greater severity on the drug use scale, less social and family support in the suescales of EUROP-ASI than compared to patients under 45 years in the group MMP-NO HRP. In conclusion, MMP-HRP patients are younger compared to MMP-NO HRP patients, they also receive higher doses of methadone and had more intravenous use. The above findings imply that the early onset of high risk drug use and long-term exposure to heroin have more severe outcomes such as higher comorbidities (e.g. infectious diseases, medical and psychiatric disorders), and consequently, these patients are a more vulnerable group with a worse prognosis.

Keywords: harm reduction program; methadone maintenance treatment; HIV, hepatitis C, heroine dependence.
\end{abstract}




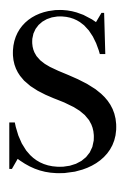
e estima que el número de consumidores de heroína en Programa de Mantenimiento con Metadona (PMM) en España es cercano a unos 65.648 usuarios (Observatorio Español de la Droga y las Toxicomanías [OEDT, 2015]). La dependencia de heroína es un problema crónico, con historia de recaídas, asociada a trastornos médicos y a otros trastornos mentales, problemática que se incrementa si se asocia al uso de heroína por vía intravenosa (Roncero et al., 2016; Miguel-Arias, Pereiro, Bermejo, López de Abajo y Sobrino, 2016).

Los programas sustitutivos con agonistas opiáceos han demostrado elevada eficacia, siendo en la actualidad los más utilizados en pacientes con dependencia de opiáceos, así como para reducir la prevalencia de infección por el VIH (Amato, Davoli, Perucci, Ferri, Faggiano y Mattick, 2005; Fernandez-Miranda, García-Portilla, Sáiz, Gutiérrez y Bobes, 2001; MacArthur et al., 2014). Existen estudios observacionales que demuestran la utilidad en relación a la capacidad de retención en tratamiento de los PMM, disminución del consumo de drogas ilegales, de las prácticas de riesgo, de la comorbilidad y morbi-mortalidad (VIH, hepatitis víricas) y criminalidad asociadas, así como la mejoría en el empleo y calidad de vida del adicto (FernándezMiranda et al., 2001; Havinga, van der Velden, de Gee y van der Poel, 2014; Salamina et al., 2010).

Hay una amplia variabilidad en la respuesta a metadona (Marie-Claire et al., 2016) y algunos pacientes pueden necesitar dosis bajas. Sin embargo, dosis de metadona por encima de 60-80 mg/día, un amplio y fácil acceso a servicios asistenciales y psicosociales, se relacionan con mayor adherencia al tratamiento y disminución del uso de drogas, aunque la comorbilidad psiquiátrica y la codependencia de sustancias ensombrecen el pronóstico (Amato et al., 2005; Fernández-Miranda et al., 2001).

Se ha descrito que en dependientes de heroína en tratamientos sustitutivos de opiáceos, un $83 \%$ de los pacientes presentan al menos una comorbilidad clínica, presentando el $69 \%$ alguna comorbilidad física y un $59 \%$ y un $40 \%$ una comorbilidad infecciosa y no infecciosa respectivamente (Roncero et al., 2011). También existe alta prevalencia de enfermedades mentales en consumidores de opiáceos (Fernández-Miranda et al., 2001; Herrero, Domingo-Salvany, Brugal, Torrens y Itinere Investigators, 2011; Roncero et al., 2016; San et al., 2016) con comorbilidad en el Eje I $(52 \%)$ y el Eje II (19\%) (Roncero, et al., 2011). El trastorno mental más frecuente es el trastorno depresivo y el trastorno de ansiedad, con una prevalencia a lo largo de la vida de entre el 4 - 54\%; la prevalencia de otros trastornos psiquiátricos mayores como la esquizofrenia, el trastorno obsesivo-compulsivo y los trastornos alimentarios, es menor (Astals, Díaz, Domingo-Salvany, Martín-Santos, Bulbena y Torrens, 2009).

Programas asociados a la intervención terapéutica en consumidores de drogas crónicos, de los cuales los depen- dientes a heroína constituyen un porcentaje importante, son los programas de reducción de daños (REDAN) (International Harm Reduction Association [IHRA], 2009). REDAN se refiere a las políticas, programas y prácticas que buscan reducir las consecuencias en salud, sociales y económicas asociadas con el uso de drogas psicoactivas, en personas que no pueden o no quieren parar el consumo (Daigre et al., 2010; IHRA, 2009). El enfoque principal es la prevención del daño, en personas que continúan usando drogas, por lo que estos programas se incluyen dentro de la prevención terciaria (Rhodes y Hedrich, 2010; Torrens, Fonseca, Castillo y Domingo-Salvany, 2013). Sus objetivos son minimizar las complicaciones médicas y psicopatológicas derivadas del consumo de drogas, promover el uso de vías menos incisivas o peligrosas, enseñar hábitos higiénico-dietéticos saludables, acercar al paciente progresivamente a los recursos asistenciales y fomentar finalmente la abstinencia de sustancias (IHRA, 2009). Un grupo especialmente vulnerable son los jóvenes en quienes se ha evidenciado un incremento continuo en las tasas de uso ilícito de drogas y muertes relacionadas por sobredosis, particularmente en los que se inyectan drogas. Existen estudios que han demostrado que ser joven, tener arrestos frecuentes y el consumo moderado/grave de alcohol con ingresos recientes para desintoxicación de metadona incrementan el riesgo de sobredosis (Seal, Kral, Gee, Moore, Bluthenthal, Lorvick, y Edlin, 2001).

Los programas REDAN contemplan medidas parciales y/o de carácter paliativo en consumidores graves o en situación de exclusión social, buscan prevenir las infecciones más prevalentes (programas de intercambio de jeringuillas y dispensación de preservativos) e incrementar el control y tratamiento de la patología orgánica y psiquiátrica asociada al consumo (acceso fácil y cercano a los servicios de salud). En España los programas de tratamiento con metadona a nivel público se iniciaron en el año 1985, sin embargo no se expandieron hasta los años 90 con un enfoque terapéutico y de REDAN. Los programas de Intercambio de Jeringuillas (PIJ) se iniciaron también alrededor de estas fechas, siendo incluidas las salas de venopunción o consumo supervisado en el año 2000 (Martínez-Luna, Palma-Álvarez y Roncero, 2016).

Los objetivos del estudio son comparar pacientes en PMM que utilizan servicios de PMM-REDAN versus pacientes en PMM-NO REDAN. Se hipotetiza que los pacientes en PMM-REDAN tienen mayor gravedad en la adicción y mayor comorbilidad en cuanto a enfermedades tanto físicas (VIH, Hepatitis C) como mentales, con más complejidad clínico-psicopatológica que los PMM-NO REDAN.

\section{Método}

El estudio se realizó en el Centro de Atención y Seguimiento (CAS) Vall d'Hebron. Los datos fueron recogidos 
desde enero de 2006 hasta enero de 2016. Los criterios de inclusión fueron: ser mayor de edad, presentar dependencia de opiáceos según criterios DSM IV-TR y DSM-5, firmar el consentimiento informado y finalizar el proceso de evaluación, cuyo protocolo previamente había sido aprobado por el Comité de Ética Hospitalaria. Los criterios de exclusión fueron: presentar signos de intoxicación, enfermedad somática grave, deterioro cognitivo grave y barrera idiomática, todo ello objetivado en la entrevista basal. Los pacientes no recibieron compensación económica por su participación en el estudio. Este trabajo forma parte de una investigación más amplia sobre comorbilidad en pacientes con trastornos por dependencia de sustancias.

De los 225 pacientes que cumplían criterios diagnósticos de trastorno por dependencia de opiáceos o trastorno por consumo moderado/grave según criterios DSM-IV-TR (años 2006-2013) y DSM-5 (años 2014-2016) y estaban en PMM, 143 pacientes terminaron el protocolo de evaluación psicológica. La muestra se dividió en dos grupos: el primer grupo estaba formado por los pacientes en PMMNO REDAN n=101 y el segundo grupo está compuesto por los pacientes en PMM-REDAN n=42; para incluirlos en este grupo habían utilizado 2 o más servicios de reducción de daños (intercambio jeringuillas, sala de consumo supervisado, taller de sobredosis).

De los 143 pacientes incluidos se realizó un subanálisis en menores de 45 años. Este subanálisis se hizo en base a un cálculo aproximado de la generación de consumidores en la que empezaron a ofrecerse los servicios REDAN de una manera generalizada en España. Se dividió la muestra entre la generación previa a REDAN (mayores de 45 años) y la generación posterior al inicio de REDAN (menores de 45 años), quedando una muestra de 116 pacientes, de los cuales 78 pacientes eran del grupo PMM-NO REDAN y 38 del grupo PMM-REDAN.

\section{Variables e Instrumentos}

Se realizaron tres entrevistas diagnósticas durante las cuales se recogieron datos sociodemográficos (sexo, edad, nacionalidad, nivel de estudios), variables relacionadas con el consumo de opiáceos (edad de inicio de la dependencia, cantidad de consumo, dosis de metadona pautada). Las evaluaciones fueron realizadas por psicólogas entrenadas. Los instrumentos utilizados fueron:

- Cuestionario sociodemográfico y clínico desarrollado ad hoc por nuestra unidad que incluye dentro de sus variables enfermedades infecciosas (VIH, Tuberculosis, etc) y hepáticas (hepatopatías, cirrosis, hepatitis infecciosas u otras hepatitis) (Grau-López et al., 2012).

- EuropASI Versión Española (Bobes, González, Saiz y Bousoño, 1996) de la Versión Europea del Addiction Severity Index (EuropASI) (Kokkevi y Hartgers, 1995). Entrevista clínica estructurada y heteroaplicada que explora las siguientes áreas: problemas médicos, situación laboral/soportes, consumo de alcohol/drogas, problemas legales, relaciones sociofamiliares y estado psicológico. Se han utilizado las puntuaciones compuestas al controlar el grado de subjetividad del entrevistador.

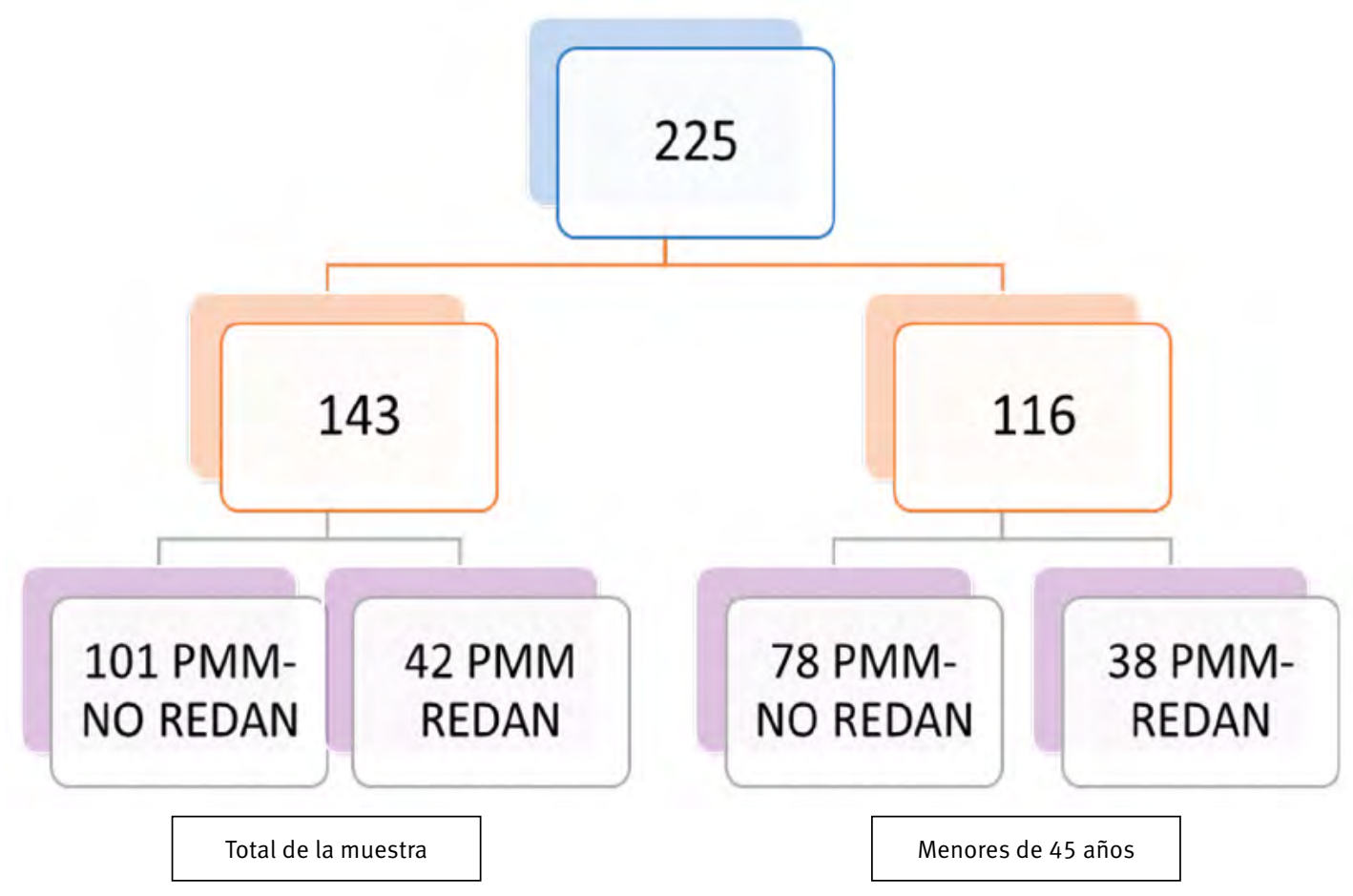

Figura 1. Flujo de pacientes del estudio. 
- SCID-I (Entrevista clínica semi-estructurada para los trastornos del eje I del DSM-IV) (First, Spitzer, Gibbon y Williams, 1999).

- SCID-II (First, Gibbon, Spitzer, Williams y Smith, 1999).

Los instrumentos se utilizaron independientemente de que el diagnóstico clínico se realizara con criterios DSMIV-TR y/o DSM-V.

\section{Análisis de datos}

En el análisis de datos se realizó análisis descriptivo, bivariante y multivariante. La descripción de todas las variables en términos de porcentajes, medias y desviaciones estándar. Para el análisis bivariante se utilizó el test de Chi Cuadrado para comparar variables categóricas y se utilizó la prueba t student de las variables continuas. Se realizó un ajuste de los resultados mediante corrección de Bonferroni. Las variables que resultaron significativas en el bivariante se utilizaron en el análisis multivariante de regresión logística. Los datos se recogieron y analizaron mediante el paquete estadístico SPSS versión 18.0.

\section{Resultados}

\section{Variables clínicas, sociodemográficas y de consumo en la muestra total}

En el análisis bivariado se encuentran diferencias en cuanto a la edad $(39.53 \pm 8.20$ vs $34.83 \pm 7.37$; $\mathrm{p} \leq .01)$, siendo más jóvenes los pacientes PMM-REDAN. En cuanto a variables clínicas tales como antecedentes médicos (enfermedades infecciosas y hepáticas) y antecedentes psiquiátricos (trastornos afectivos, de personalidad, psicóticos y de ansiedad) no se evidenciaron diferencias significativas.

En relación a las variables de consumo, se observa que el $56.3 \%$ de los pacientes de la muestra consumen por vía endovenosa. En cuanto a la vía fumada se encuentra que los pacientes PMM-NO REDAN la utilizan más que los pacientes PMM-REDAN (22.3\% vs $4.9 \%$; $\leq .01$ ). Respecto a la dosis de metadona pautada, el $62.7 \%$ de los pacientes PMM-NO REDAN tienen dosis inferiores a $40 \mathrm{mg}$ frente al 41,7 \% del grupo PMM-REDAN ( $\mathrm{p} \leq .046$ ), aunque no sigue siendo estadísticamente significativo después de la corrección de Bonferroni.

\section{Variables clínicas, sociodemográficas y de consumo en los menores de 45 años}

$\mathrm{Al}$ hacer un subanálisis por edad en el grupo de menores de 45 años, se puede observar que los pacientes PMM-REDAN son más jóvenes $(36.54 \pm 6.10$ vs $33.42 \pm 6.19$; p $\leq .05)$ y un $58,1 \%$ de los pacientes PMM-REDAN presentan enfermedades hepáticas. En relación a estas enfermedades, en el total de la muestra, se realizó un subanálisis en los pacientes mayores de 45 años, donde existe una diferencia significativa en la presencia de enfermedades hepáticas en los pacientes PMM-REDAN (.035). Al comparar los pacientes PMM-REDAN se detectó que el grupo PMM-REDAN de menores de 45 años presentaba menos prevalencia de enfermedades hepáticas.

Respecto a las variables de consumo, los pacientes PMM-REDAN utilizan más la vía intravenosa $(5.9 \%$ vs 70.3 $\%$; $\leq .05)$, mientras que el grupo PMM-NO REDAN presentan mayor uso de la vía fumada $(24.3 \%$ vs $5.4 \%$; $\leq .05)$.

Tabla 1. Variables sociodemográficas y clínicas de la muestra total.

\begin{tabular}{|c|c|c|c|c|c|}
\hline VARIABLES SOCIODEMOGRÁFICAS & Total (143) M DDS & PMM-NO REDAN (101) MEDS & PMM-REDAN (42) MEDS & & \\
\hline Edad (años) & $\begin{array}{c}38.13 \pm 8.22 \\
\%\end{array}$ & $\begin{array}{c}39.53 \pm 8.20 \\
\%\end{array}$ & $\begin{array}{c}34.83 \pm 7.37 \\
\%\end{array}$ & $\begin{array}{c}3.199 \\
\mathbf{X}^{2}\end{array}$ & $\begin{array}{c}<.01^{\star} \\
\mathbf{P}\end{array}$ \\
\hline Mayores de 45 años & 22 & 25.3 & 14.3 & 2.07 & .150 \\
\hline Género (hombre) & 69.7 & 73 & 61.9 & 1.725 & .189 \\
\hline Estado civil (casado) & 27.4 & 25.5 & 31.7 & .054 & .816 \\
\hline Situación laboral (activos) & 20.7 & 22.3 & 17.0 & .482 & .488 \\
\hline Estudios superiores (>Bachillerato) & 46 & 47.9 & 41.5 & .482 & .575 \\
\hline VARIABLES CLÍNICAS & $\%$ & $\%$ & $\%$ & $\mathbf{X}^{2}$ & $\mathbf{P}$ \\
\hline Antecedentes Médicos & 70.8 & 70.1 & 72.5 & .079 & .839 \\
\hline Enfermedades infecciosas & 40.9 & 40.2 & 42.5 & .098 & .952 \\
\hline Enfermedades hepáticas & 43 & 38 & 55.6 & 3.440 & .179 \\
\hline Antecedentes Psiquiátricos & 54 & 54.6 & 52.5 & .052 & .852 \\
\hline Trastornos Afectivos & 20.6 & 22.9 & 15 & 1.415 & .493 \\
\hline Trastornos de Personalidad & 25.7 & 21.9 & 35 & 2.629 & .269 \\
\hline Trastornos de Ansiedad & 9.4 & 8.8 & 11.1 & .320 & .852 \\
\hline Trastorno Psicótico & 6.6 & 6.3 & 7.5 & .281 & .869 \\
\hline Trastorno psicótico inducido & 54.2 & 54.8 & 52.8 & .040 & .845 \\
\hline Autoreferencia & 30.8 & 31.5 & 29.4 & .048 & 1 \\
\hline Delirios persecución & 28.3 & 31.9 & 20.6 & 1.468 & .257 \\
\hline Alucinaciones & 28.3 & 30.6 & 23.5 & .562 & .498 \\
\hline
\end{tabular}

Nota. PMM-NO REDAN= Programa de Mantenimiento con Metadona, no Reducción de Daños; REDAN= Reducción de daños. M=Media; DS=Desviación Estándar; t= T-Student.

*(corrección Bonferroni). 
Nieves Martínez-Luna, Laia Rodriguez-Cintas, Abderraman Esojo, Raúl Felipe Palma-Álvarez, María Robles-Martínez, Lara Grau-López, Marta Perea, Carlos Roncero

\section{Comorbilidad psiquiátrica}

No se encuentran diferencias en cuanto a la comorbilidad psiquiátrica en el eje I, en ninguno de los análisis realizados en este estudio. Se encuentra diferencia esta- dísticamente significativa en cuanto a los trastornos de personalidad del clúster $\mathrm{B}$, sin embargo esta diferencia no permanece estadísticamente significativa después de la corrección de Bonferroni.

Tabla 2. Variables relacionadas con el consumo de la muestra total.

\begin{tabular}{|c|c|c|c|c|c|}
\hline VARIABLES RELACIONADAS CON EL CONSUMO & Total (143) M $\mathrm{DSS}$ & PMM-NO REDAN (101) M $\pm D S$ & PMM-REDAN (42) M \pm DS & $\mathbf{t}$ & p \\
\hline Edad de inicio del consumo de opiáceos & $20.52 \pm 6.60$ & $21 \pm 7.12$ & $19.43 \pm 5.15$ & 1.263 & .209 \\
\hline Edad de inicio dependencia de opiáceos & $22.42 \pm 7.52$ & $22.76 \pm 7.70$ & $21.73 \pm 7.18$ & .717 & .475 \\
\hline Años de consumo de opiáceos & $11.60 \pm 8.65$ & $11.57 \pm 8.22$ & $11.64 \pm 9.62$ & .034 & .973 \\
\hline Cantidad opiáceos 6 meses (gr/semana) & $.669 \pm 1.30$ & $.701 \pm 1.40$ & $.602 \pm 1.08$ & .398 & .631 \\
\hline Cantidad opiáceos último mes (gr/semana) & $.655 \pm 1.21$ & $.694 \pm 1.27$ & $.580 \pm 1.10$ & .486 & .628 \\
\hline OTROS TUS & $\%$ & $\%$ & $\%$ & $\mathbf{X}^{2}$ & $\mathbf{P}$ \\
\hline Dependencia de Alcohol & 37.8 & 40.6 & 31 & 1.173 & .345 \\
\hline Dependencia de Cannabis & 30.1 & 29.7 & 31 & .022 & 1 \\
\hline Dependencia de Benzodicepinas & 29.4 & 26.7 & 35.7 & 1.154 & .316 \\
\hline Dependencia de Cocaína & 57.7 & 57 & 59.5 & .077 & .853 \\
\hline Dependencia de Tabaco & 84.7 & 87.5 & 78 & 1.977 & .196 \\
\hline Politoxicomanía & 47.2 & 52.8 & 47.2 & 1.977 & .198 \\
\hline Vía principal de consumo de opiáceos & $\%$ & $\%$ & $\%$ & $\mathbf{X}^{2}$ & p \\
\hline Intranasal & 18.5 & 18.1 & 19.5 & .039 & .815 \\
\hline Intravenosa & 56.3 & 51.1 & 68.3 & 3.444 & .089 \\
\hline Inhalada & 3 & 2.1 & 4.9 & Ns & ns \\
\hline Fumada & 17 & 22.3 & 4.9 & 6.159 & $<.01^{*}$ \\
\hline Otras & 5.2 & 6.4 & 2.4 & Ns & ns \\
\hline METADONA & $M \pm D S$ & $M \pm D S$ & $M \pm D S$ & $\mathbf{T}$ & $\mathbf{P}$ \\
\hline \multirow[t]{2}{*}{ Media dosis metadona } & $52.48 \pm 48.59$ & $46.86 \pm 51.30$ & $61.69 \pm 42.91$ & 1.452 & .150 \\
\hline & $\%$ & $\%$ & $\%$ & $\mathrm{X}^{2}$ & $P$ \\
\hline Metadona Baja («40) & 54.7 & 62.7 & 41.7 & 3.997 & $<.05$ \\
\hline Metadona Media (40-80) & 29.5 & 25.4 & 36.6 & 1.229 & .268 \\
\hline Metadona Alta (>80) & 15.8 & 11.9 & 22.2 & 1.804 & .179 \\
\hline
\end{tabular}

Nota. PMM-NO REDAN= Programa de Mantenimiento con Metadona, no Reducción de Daños; REDAN= Reducción de daños. $M$ =Media; $\mathrm{DS}=$ Desviación Estándar; $\mathrm{t=}$ T-Student; $\mathrm{X}^{2}=$ Chi-Cuadrado; $\mathrm{MI}=$ Muestra Insuficiente; TUS= Trastorno por Uso de Sustancias. ns: no prueba estadística, muestra insuficiente.

a Consumidor de opiáceos más otras dos sustancias tóxicas diferentes.

*(corrección Bonferroni)

Tabla 3. Variables sociodemográficas y clínicas de la muestra de menores de 45 años.

\begin{tabular}{|c|c|c|c|c|c|}
\hline VARIABLES SOCIODEMOGRÁFICAS & Total (116) M $\pm \mathrm{DS}$ & PMM-NO REDAN (78) M \pm DS & PMM-REDAN (38) M $\pm D S$ & $\mathbf{t}$ & $\mathbf{P}$ \\
\hline Edad (años) & $\begin{array}{c}35.52 \pm 6.28 \\
\%\end{array}$ & $\begin{array}{c}36.54 \pm 6.10 \\
\%\end{array}$ & $\begin{array}{c}33.42 \pm 6.19 \\
\%\end{array}$ & $\begin{array}{c}2.572 \\
\mathbf{X}^{2}\end{array}$ & $\begin{array}{c}<.05^{\star} \\
\mathbf{P}\end{array}$ \\
\hline Género (hombre) & 68.1 & 71.8 & 60.5 & 1.494 & .289 \\
\hline Estado civil (casado) & 29.7 & 28.4 & 32.4 & 1.02 & .421 \\
\hline Situación laboral (activos) & 12.5 & 12 & 13.5 & .233 & .418 \\
\hline Estudios superiores (>Bachillerato) & 44.2 & 46.1 & 40.5 & .306 & .687 \\
\hline VARIABLES CLÍNICAS & $\%$ & $\%$ & $\%$ & $\mathbf{X}^{2}$ & $\mathbf{P}$ \\
\hline Antecedentes Médicos & 67.3 & 64.9 & 72.2 & .592 & .522 \\
\hline Enfermedades infecciosas & 38.1 & 35.5 & 45.7 & 1.351 & .509 \\
\hline Enfermedades hepáticas & 40.4 & 38.8 & 58.1 & 5.562 & .053 \\
\hline Antecedentes Psiquiátricos & 56.6 & 57.1 & 55.6 & .025 & 1 \\
\hline Trastornos Afectivos & 20.5 & 23 & 17.1 & .487 & .784 \\
\hline Trastornos de Personalidad & 27.7 & 23 & 40 & 3.385 & .184 \\
\hline Trastornos de Ansiedad & 9.7 & 8.7 & 12.9 & ns & ns \\
\hline Trastorno Psicótico & 7.1 & 6.8 & 8.6 & ns & ns \\
\hline Trastorno Psicótico inducido & 59.4 & 60.9 & 56.3 & .194 & .666 \\
\hline Autoreferencia & 31.8 & 32.7 & 30 & .067 & 1 \\
\hline Delirios persecución & 31 & 35.2 & 23.3 & 1.268 & .190 \\
\hline Alucinaciones & 31 & 33.3 & 26.7 & .401 & .626 \\
\hline
\end{tabular}

Nota. PMM-NO REDAN= Programa de Mantenimiento con Metadona, no Reducción de Daños; REDAN= Reducción de daños. M=Media; DS=Desviación Estándar; t= T-Student. ns: no prueba estadística, muestra insuficiente.

*(corrección Bonferroni). 
Tabla 4. Variables relacionadas con el consumo de la muestra de menores de 45 años.

\begin{tabular}{|c|c|c|c|c|c|}
\hline VARIABLES RELACIONADAS CON EL CONSUMO & Total (116) M $\mathrm{DSS}$ & PMM-NO REDAN (78) M \pm DS & PMM-REDAN (38) M $\pm D S$ & $\mathbf{T}$ & $\mathbf{P}$ \\
\hline Edad de inicio del consumo de opiáceos & $20.27 \pm 5.89$ & $20.92 \pm 6.27$ & $19 \pm 4.89$ & 1.754 & .083 \\
\hline Edad de inicio dependencia de opiáceos & $22.62 \pm 6.78$ & $22.70 \pm 7.11$ & $20.81 \pm 6.04$ & 1.425 & .158 \\
\hline Años de consumo de opiáceos & $10.49 \pm 8.10$ & $10.16 \pm 7.50$ & $11.06 \pm 9.14$ & .474 & .637 \\
\hline Cantidad opiáceos 6 meses (gr/semana) & $.691 \pm 1.39$ & $.729 \pm 1.51$ & $.620 \pm 1.13$ & .387 & .700 \\
\hline Cantidad opiáceos último mes (gr/semana) & $.677 \pm 1.27$ & $.722 \pm 1.35$ & $.602 \pm 1.15$ & .449 & .654 \\
\hline OTROS TUS & $\%$ & $\%$ & $\%$ & $\mathbf{X}^{2}$ & $\mathbf{P}$ \\
\hline Dependencia de Alcohol & 38.7 & 41 & 34.2 & .500 & .546 \\
\hline Dependencia de Cannabis & 31.9 & 33.3 & 28.9 & .226 & 6.77 \\
\hline Dependencia de Benzodicepinas & 31 & 26.9 & 39.5 & 1.888 & .124 \\
\hline Dependencia de Cocaína & 60.3 & 61.5 & 57.9 & .142 & .840 \\
\hline Dependencia de Tabaco & 85.8 & 88.2 & 81.1 & 1.025 & .390 \\
\hline Politoxicomanía & 49.1 & 53.8 & 39.5 & 2.112 & .169 \\
\hline Vía principal de consumo de opiáceos & $\%$ & $\%$ & $\%$ & $\mathbf{X}^{2}$ & $\mathbf{P}$ \\
\hline Intranasal & 18.9 & 20.3 & 16.2 & .264 & .406 \\
\hline Intravenosa & 54.1 & 5.9 & 70.3 & 5.876 & $<.05^{*}$ \\
\hline Inhalada & 3.6 & 2.7 & 5.4 & .519 & .407 \\
\hline Fumada & 18 & 24.3 & 5.4 & 5.977 & $<.05^{*}$ \\
\hline Otras & 18.9 & 20.3 & 16.2 & .264 & .406 \\
\hline METADONA & $\mathrm{M} \pm \mathrm{DS}$ & $\mathrm{M} \pm \mathrm{DS}$ & $\mathrm{M} \pm \mathrm{DS}$ & $\mathbf{T}$ & $\mathbf{P}$ \\
\hline Media dosis metadona & $\begin{array}{c}55.30 \pm 47.20 \\
\%\end{array}$ & $\begin{array}{c}48.17 \pm 48.78 \\
\%\end{array}$ & $\begin{array}{c}64.66 \pm 44.05 \\
\%\end{array}$ & $\begin{array}{c}1.502 \\
X^{2}\end{array}$ & $\begin{array}{c}.138 \\
P\end{array}$ \\
\hline Metadona Baja (८40) & 50 & 59.5 & 37.5 & 3.524 & .100 \\
\hline Metadona Media (40-80) & 31.1 & 26.2 & 37.5 & 1.084 & .322 \\
\hline Metadona Alta $(>80)$ & 18.9 & 14.3 & 25 & 1.359 & .369 \\
\hline
\end{tabular}

Nota. PMM-NO REDAN= Programa de Mantenimiento con Metadona, no Reducción de Daños; REDAN= Reducción de daños. M=Media; DS=Desviación Estándar; t= T-Student; $\mathrm{X}^{2}=$ Chi-Cuadrado; $\mathrm{MI}=$ Muestra Insuficiente; TUS= Trastorno por Uso de Sustancias.

a Consumidor de opiáceos más otras dos sustancias diferentes.

*(corrección Bonferroni)

Tabla 5. Trastornos Mentales según entrevistas diagnósticas SCID I y SCID II

\begin{tabular}{|c|c|c|c|c|c|}
\hline Muestra total & $\begin{array}{c}\text { Total (143) } \\
\%\end{array}$ & $\begin{array}{c}\text { PMM-NO REDAN (101) } \\
\%\end{array}$ & $\begin{array}{c}\text { PMM-REDAN (42) } \\
\%\end{array}$ & $\mathbf{X}^{2}$ & $\mathbf{P}$ \\
\hline Trastorno estado animo inducido & 23.4 & 21 & 29.3 & 1.109 & .381 \\
\hline Trastorno ansiedad inducido & 7.7 & 5.9 & 11.9 & Ns & Ns \\
\hline Clúster A & 4.2 & 5 & 2.4 & Ns & Ns \\
\hline Clúster B & 45.4 & 36 & 54.8 & 4.287 & $<.05$ \\
\hline Clúster C & 6.3 & 6 & 7.1 & Ns & Ns \\
\hline Menores de 45 años & $\begin{array}{c}\text { Total (116) } \\
\%\end{array}$ & $\begin{array}{c}\text { PMM-NO REDAN (78) } \\
\%\end{array}$ & $\begin{array}{c}\text { PMM-REDAN (38) } \\
\%\end{array}$ & $\mathbf{X}^{2}$ & $\mathbf{p}$ \\
\hline Trastorno estado animo inducido & 22.8 & 19.5 & 29.7 & 1.491 & .240 \\
\hline Trastorno ansiedad inducido & 9.5 & 7.7 & 13.2 & ns & Ns \\
\hline Clúster A & 4.3 & 5.1 & 2.6 & ns & Ns \\
\hline Clúster B & 44.8 & 38.5 & 57.9 & 3.902 & $<.05$ \\
\hline Clúster C & 6 & 5.1 & 7.9 & ns & Ns \\
\hline
\end{tabular}

Nota. PMM-NO REDAN= Programa de Mantenimiento con Metadona, no Reducción de Daños; REDAN= Reducción de daños. M=Media; DS=Desviación Estándar; t=

T-Student. ns: no prueba estadística, muestra insuficiente.

*(corrección Bonferroni).

Finalmente, al estudiar los resultados obtenidos en la entrevista EuropASI se objetivó que, en el total de la muestra, únicamente existen diferencias significativas

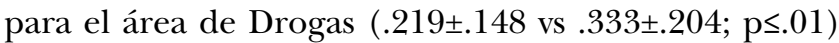
siendo más severo en el grupo PMM-REDAN.

$\mathrm{Al}$ analizar los resultados obtenidos para la muestra de menores de 45 años se encuentra que en la subescala de drogas $(.210 \pm .159$ vs $.332 \pm .206 ; \mathrm{p} \leq .01)$ y familiar (.294 \pm .292 vs $.433 \pm .341 ; \mathrm{p} \leq .05)$ presentan diferencias estadísticamente significativas. En la subescala de dro- gas se detecta mayor gravedad en el consumo de drogas en el grupo PMM-REDAN, al igual que en la subescala familiar, lo que implica mayores problemas de consumo y peor soporte familiar en los pacientes PMM-REDAN.

\section{Análisis Multivariante}

Se realizó un análisis de regresión logística para detectar las variables relacionadas con la gravedad en el grupo PMM-REDAN, con el fin de analizar todas las variables del análisis bivariante previo que permanecían estadísticamen- 
Tabla 6. Gravedad de la Adicción (EuropASI).

\begin{tabular}{|c|c|c|c|c|c|}
\hline \multicolumn{6}{|c|}{ Total muestra } \\
\hline EuropASI & total & PMM-NO REDAN & PMM-REDAN & $\mathrm{t}$ & p \\
\hline Médica & $.323 \pm .356$ & $.348 \pm .364$ & $.261 \pm .333$ & 1.320 & .189 \\
\hline Laboral & $.612 \pm .288$ & $.597 \pm .289$ & $.651 \pm .294$ & 1.018 & .310 \\
\hline Alcohol & $.175 \pm .235$ & $.175 \pm .238$ & $.173 \pm .228$ & .037 & .970 \\
\hline Drogas & $.253 \pm .173$ & $.219 \pm .148$ & $.333 \pm .204$ & 3.271 & $<.01$ * \\
\hline Legal & $.161 \pm .245$ & $.167 \pm .250$ & $.148 \pm .237$ & .428 & .670 \\
\hline Familiar & $.343 \pm .314$ & $.318 \pm .300$ & $.402 \pm .343$ & 1.476 & .142 \\
\hline Psicológica & $.331 \pm .234$ & $.327 \pm .247$ & $.341 \pm .202$ & .318 & .751 \\
\hline \multicolumn{6}{|c|}{ Menores de 45 años } \\
\hline EuropASI & total $<45$ & PMM-NO REDAN & PMM-REDAN & $\mathbf{t}$ & $\mathbf{P}$ \\
\hline Médica & $.301 \pm .345$ & $.321 \pm .351$ & $.261 \pm .333$ & .865 & .381 \\
\hline Laboral & $.617 \pm .295$ & $.599 \pm .289$ & $.655 \pm .307$ & .952 & .354 \\
\hline Alcohol & $.167 \pm .225$ & $.163 \pm .220$ & $.176 \pm .237$ & .300 & .771 \\
\hline Drogas & $.250 \pm .184$ & $.210 \pm .159$ & $.332 \pm .206$ & 3.213 & $<.01^{*}$ \\
\hline Legal & $.169 \pm .251$ & $.175 \pm .255$ & $.157 \pm .245$ & .356 & .719 \\
\hline Familiar & $.340 \pm .314$ & $.294 \pm .292$ & $.433 \pm .341$ & 2.277 & $<.05^{\star}$ \\
\hline Psicológica & $.334 \pm .236$ & $.325 \pm .254$ & $.354 \pm .197$ & .677 & .500 \\
\hline
\end{tabular}

Nota. PMM-NO REDAN= Programa de Mantenimiento con Metadona, no Reducción de Daños; REDAN= Reducción de daños. M=Media; DS=Desviación Estándar;

$\mathrm{t}=$ T-Student. ns: no prueba estadística, muestra insuficiente.

*(corrección Bonferroni).

te significativas después de la corrección de Bonferroni. Para el total de la muestra, estas variables fueron: edad, vía fumada y gravedad de la adicción en la subescala drogas. Las tres variables se mantuvieron como factores significativos en el modelo.

Para la muestra de menores de 45 años, estas variables fueron: edad, vía intravenosa, vía fumada y gravedad de la adicción en las subescalas de drogas y familiar. Solo se mantuvieron como factores significativos en el modelo, la edad, la vía fumada y la gravedad de adicción de la subescala de drogas, se excluyeron del modelo la vía intravenosa y la gravedad de la adicción de la subescala familiar.

Tabla 7. Análisis multivariante ajustando el efecto de las variables significativamente asociadas en el análisis bivariado del estudio.

\begin{tabular}{lcccc}
\hline \multicolumn{5}{c}{ Total de la muestra } \\
\hline Variables & Wald & $\mathbf{p}$ & $\mathbf{0 . R}$. & $\mathbf{9 5 \%}$ IC \\
\hline Edad & 8.87 & .003 & .92 & $.87-.97$ \\
Vía fumada & 5.74 & .017 & 44.66 & $4.04-494.17$ \\
EuropASI drogas & 9.59 & .002 & .14 & $.03-.70$ \\
\hline \multicolumn{5}{c}{ Menores de 45 años* } \\
\hline Variables & Wald & $\mathbf{p}$ & $\mathbf{0 . R}$. & $\mathbf{9 5 \%}$ IC \\
\hline Edad & 4.97 & .03 & .92 & $.86-.99$ \\
Vía fumada & 5.35 & .02 & .15 & $.03-.75$ \\
EuropASI drogas & 8.61 & .00 & 40.36 & $3.42-476.84$ \\
\hline
\end{tabular}

Nota. *Variable eliminada en el paso 2: EuropASI familiar.

Nota. *Variable eliminada en el paso 3: Vía intravenosa.

\section{Discusión}

Existen diferencias entre los pacientes en PMM que utilizan servicios de Reducción de daños (PMM-REDAN) y los pacientes en PMM que no los utilizan (PMM-NO RE-
DAN). Los pacientes PMM-REDAN son más jóvenes. En el momento actual no existen estudios que relacionan específicamente este dato, aunque algunos trabajos señalan la importancia de la edad ya que al ser más jóvenes presentan menor adherencia en los programas REDAN y mayor dificultad para acceder a servicios del mismo tipo (Krug, Hildebrand y Sun, 2015). El inicio más temprano y el mayor tiempo de exposición a las prácticas de riesgo de consumo del grupo PMM-REDAN se traducirían en mayor riesgo a presentar comorbilidad médica y psiquiátrica (Hopfer, Khuri, Crowley y Hooks, 2002). En los pacientes PMM-REDAN se detecta más presencia de enfermedades hepáticas (55.6\% vs 38\%, .053), además en este grupo los pacientes mayores de 45 años presentan diferencia significativa (.035), lo que ya se ha descrito en la literatura acerca de pacientes que hacen uso de programas de intercambio de jeringuillas o salas de consumo, quienes tienen altas prevalencias de enfermedad mental, VIH y Hepatitis C (Havinga et al., 2014; Herrero, Domingo Salvany, Brugal, Torrens y Itinere Investigators, 2011; Wang, Zhang y Ho, 2011).

Aunque la existencia de trastornos del clúster B en los pacientes en PMM es esperable (Paversen, Masoudi, Majidi-Tabrizi y Mazhari, 2012; Roncero et al., 2016), más de la mitad del grupo PMM-REDAN tiene alguno de estos trastornos, lo que se puede interpretar como un marcador de gravedad, ya que los trastornos de personalidad en PMM se han asociado con un peor pronóstico (Peles, Schreiber, Domany y Adelson, 2014). Sin embargo, dado que las diferencias no se mantienen se debe ser cauto en la interpretación de los trastornos de personalidad.

La existencia de trastornos mentales en pacientes de PMM se ha asociado a peor calidad de vida (Teoh Bing Fei, Yee y Habil, 2016), aunque no hay diferencias entre los dos 
grupos, se debe destacar que en el total de la muestra existe alta prevalencia de otros trastornos mentales, confirmando los hallazgos previos en dependientes de heroína en PMM (Paversen et al., 2012, Pereiro et al., 2013; Roncero et al., 2016), todo ello reflejaría que el consumo de opiáceos es grave en sí independientemente del uso de recursos REDAN.

Clínicamente se debe destacar que más de la mitad de la muestra estudiada han presentado trastornos psicóticos inducidos, sin que existan diferencias entre los grupos PMM-REDAN y PMM-NO REDAN. La presencia de psicosis en estos pacientes no debe extrañar, ya que cerca del $60 \%$ presentan también dependencia de cocaína, hecho que ha sido ampliamente asociado con la presencia de síntomas psicóticos (Roncero et al, 2017), especialmente en el grupo de autoinyectores de cocaína que reciben dosis bajas de metadona (Roncero et al, 2013b). Por otra parte, la presencia de síntomas psicóticos en adictos es una variable que incide en mayor gravedad y comorbilidad con otros trastornos mentales (Roncero et al, 2017; Roncero et al, 2013a).

Con respecto a la vía de consumo ya es sabido que los pacientes que utilizan los recursos REDAN tienden a tener más consumo por vía intravenosa independientemente de la droga de consumo (MacArthur et al., 2014), por eso el intercambio de jeringuillas y las salas de consumo supervisado por vía intravenosa fueron los programas iniciales y fundadores de REDAN (IHRA, 2010). Nuestros hallazgos corroboran este hecho al identificarse mayor uso de la vía intravenosa $(5.9 \%$ vs $70.3 \%, .017)$ en el grupo PMM-REDAN, mientras que el grupo PMM-NO REDAN presenta mayor uso de la vía fumada $(24.3 \%$ vs $5.4 \%, .011)$. La vía de consumo es importante por los riesgos que en sí conlleva, ya que existe una mayor exposición al contagio de enfermedades infecciosas (hepatitis B y C y VIH) y de las complicaciones médicas que se derivan de dichas enfermedades (Palmateer, Kimber, Hickman, Hutchinson, Rhodes y Goldberg, 2010). Por ello son necesarias estrategias para disminuir el riesgo de contagio y la morbi-mortalidad asociadas (MacArthur et al., 2014).

En relación a la dosis de metadona cabe destacar que el grupo PMM-REDAN tiene dosis más altas de metadona en comparación con el PMM-NO REDAN que presenta dosis más bajas (menores de $40 \mathrm{mg}$ /día), aún así en ambos grupos el intervalo de dosis es baja en relación a las dosis sugeridas en la literatura (Faggiano, Vigna-Taglianti, Versino, y Lemma, 2003). Las dosis bajas de metadona en el grupo PMM-REDAN se asocian a lo descrito sobre una población con un perfil de reducción de daños donde la abstinencia no es el objetivo principal, encuadrando la dosis dentro de un programa con bajo nivel de exigencia, típico de los PMM de bajo umbral (Kwan, Wong y Lee, 2015). Se puede hipotetizar que las dosis bajas de metadona en el grupo PMM-NO REDAN, se relacionan con la resistencia por parte de los pacientes de mantener dosis por encima de los 60 mg y por el temor a la retirada total de la metadona.
Cuando se valora la gravedad del consumo en el grupo PMM-REDAN, mediante la entrevista EuropASI, se objetiva que en el total de la muestra existen diferencias en la subescala de Drogas siendo más grave en el grupo PMM-REDAN. En estudios previos ya se menciona el hecho de que los pacientes REDAN en general se encuentran en un período más grave de la adicción (McNeil, Kerr, Pauly, Wood y Small, 2016). Además en la muestra de menores de 45 años se detectan diferencias tanto en la subescala de drogas como en la familiar, lo que apuntaría a menor soporte social en pacientes PMM-REDAN (Jhanjee, Lal, Mondal y Jain, 2011). Este hecho evidencia la importancia de los servicios REDAN en pacientes jóvenes al ser especialmente graves y tener menor soporte social.

Tal y como se constata en el análisis de regresión, los pacientes PMM-NO REDAN usan otras vías de consumo (fumada) más frecuentemente, lo que supondría un menor riesgo, y ya no parece tan relevante la falta de soporte familiar. Con estos resultados se puede inferir que el grupo de PMM-REDAN presenta diferencias en cuanto a variables como la edad, la vía de consumo y mayor gravedad de consumo según el EuropASI (subescala de drogas); por lo tanto se confirman los resultados de estudios previos que señalan que los pacientes que utilizan servicios de REDAN son un grupo con mayor vulnerabilidad tanto a nivel sanitario como social (Havinga et al., 2014; IHRA, 2009). Aunque se esperaban encontrar más diferencias a nivel médico y psiquiátrico entre los dos grupos, las diferencias encontradas son importantes ya que estas variables son relevantes y demuestran la mayor gravedad del grupo PMM-REDAN, información que hasta nuestro conocimiento no se ha descrito con anterioridad en España.

En relación a las limitaciones del estudio, están relacionadas con el tamaño de la muestra, lo que se explica por la complejidad que existe para poder realizar una evaluación completa en los pacientes REDAN. Otra limitación es la dosis de metadona que reciben los pacientes ya que no se especifica en que etapa del PMM estaban los pacientes (inducción, mantenimiento, retirada). Además nuestros datos son poco comparables por la falta de trabajos previos que estudien diferencias entre pacientes que utilizan los PMM-REDAN y los PMM-NO REDAN. Finalmente se debe considerar que los programas REDAN son una herramienta terapéutica y de apoyo psicosocial para pacientes graves, siendo además valorados positivamente por parte de los propios usuarios (Daigre et al., 2010), puesto que complementan la oferta asistencial dirigida a pacientes que no buscan o no pueden llegar a la abstinencia total.

\section{Reconocimientos}

Elizabeth Monterde Ochoa y Thais Ballabriga (Educadoras Sociales CAS Vall d'Hebron), Eduardo Castrillo, Miguel Angel Cantillo y Nuria Voltes Manils (Enfermeros 
CAS Vall d'Hebron), Elena Ros-Cucurull (Psiquiatra), Constanza Daigre (Psicóloga) y a todo el equipo del programa de Opiáceos y de Reducción de Daños del CAS Vall d' Hebron, ASPB (Agencia de Salut Publica de Barcelona).

\section{Conflictos de intereses}

-Dra. Nieves Martínez-Luna, declara que no tiene conflicto de intereses para el presente trabajo, pero en los últimos años ha colaborado con Janssen-Cilag, Lundbeck y Servier.

-Laia Rodriguez-Cintas declara que no tiene conflicto de intereses para el presente trabajo, pero en los últimos años ha recibido financión como colaboradora en proyectos de Jannsen-Cilag y Laboratorios Servier.

-Dr. Abderramán Esojo, declara que no tiene conflicto de intereses para el presente trabajo.

-Dr. Raúl Felipe Palma-Álvarez, declara que no tiene conflicto de interés para este trabajo, pero que en los últimos meses ha colaborado como ponente en Mundipharma.

-Dra. María Robles-Martínez declara que no tiene conflicto de intereses para el presente trabajo.

-Dra. Lara Grau-López declara que no tiene conflicto de intereses para el presente trabajo, pero en los últimos años ha colaborado con Janssen-Cilag, Lundbeck y Servier.

-Marta Perea declara que no tiene conflicto de intereses para el presente trabajo.

-Dr. Carlos Roncero declara que no tiene conflicto de intereses para el presente trabajo, pero en los últimos años ha recibido financiación como ponente y ha colaborado en proyectos de Janssen-Cilag, Pfizer, Reckitt Benckiser/ Indivior, Lundbeck, Servier, GSK, Rovi, Ferrer-Brainfarma y Astra España. Ha recibido honorarios por su participación como miembro de boards de las compañías Reckitt Benckiser/Indivior, Janssen-Cilag, Gilead y MSD. Ha dirigido el proyecto PROTEUS que fue financiado con una beca de Indivior.

\section{Referencias}

Amato, L., Davoli, M., Perucci, C. A., Ferri, M., Faggiano, F. y Mattick, R. P. (2005). An over view of systematic reviews of the effectiveness of opiate maintenance therapies: available evidence to inform clinical practice and research. Journal of Substance Abuse Treatment, 28, 321329. doi:10.1016/j.jsat.2005.02.007.

Astals, M., Díaz, L., Domingo-Salvany, A., Martín-Santos, R., Bulbena, A. y Torrens, M. (2009). Impact of co-occurring psychiatric disorders on retention in a methadone maintenance program: an 18-month follow-up study. International Journal of Environmental Research and Public Health, 6, 2822-2832. doi:10.3390/ijerph6112822.

Bobes, J., González, M. P., Saiz, P. A. y Bousoño, M. (1996). Índice Europeo de Severidad de la Adicción: EuropASI.
Versión española. Actas de la IV Reunión Interregional de Psiquiatría, 201-218.

Daigre, C., Comín, M., Rodríguez-Cintas, L., Voltes, N., Alvarez, A., Roncero, C., ... Casas, M. (2010). Users' perception of a harm reduction program in an outpatient drug dependency treatment center. Gaceta Sanitaria / S.E.S.P.A.S, 24, 446-452. doi:10.1016/j.gaceta.2010.09.007.

Faggiano, F., Vigna-Taglianti, F., Versino, E., y Lemma, P. (2003). Methadone maintenance at different dosages for opioid dependence. The Cochrane Database of Systematic Reviews, (3). doi:10.1002/14651858.CD002208.

Fernández Miranda, J., González García-Portilla, M., Sáiz Martínez, P., Gutiérrez Cienfuegos, E. y Bobes García, J. (2001). Influence of psychiatric disorders in the effectiveness of a long-term methadone maintenance treatment. Actas Españolas de Psiquiatría, 29, 228-232.

First, M.B., Spitzer, R.L., Gibbon, M. y Williams, J.B.W. (1999). Entrevista clínica estructurada para los trastornos del eje I del DSM-IV. Barcelona: Masson.

First, M.B., Gibbon, M., Spitzer, R.L., Williams, J.B.W. y Smith, B. (1999). Entrevista Clínica Estructurada para los Trastornos de la Personalidad del Eje II del DSMIV. Barcelona: Masson.

Grau-López, L., Roncero, C., Daigre, C., Gonzalvo, B., Bachiller, D., Rodriguez-Cintas, L. ... Casas, M. (2012). Risk factors of relapse in drug dependent patients after hospital detoxification. Adicciones, 24, 115-122.

Havinga, P., van der Velden, C., de Gee, A. y van der Poel, A. (2014). Differences in sociodemographic, drug use and health characteristics between never, former and current injecting, problematic hard-drug users in the Netherlands. Harm Reduction Journal, 11, 6. doi:10.1186/14777517-11-6.

Herrero, M. J., Domingo-Salvany, A., Brugal, M. T., Torrens, M. y Itinere Investigators. (2011). Incidence of psychopathology in a cohort of young heroin and/or cocaine users. Journal of Substance Abuse Treatment, 41, 55-63. doi:10.1016/j.jsat.2011.01.013.

Hopfer, C.J., Khuri, E., Crowley, T.J. y Hooks, S. (2002). Adolescent heroin use: a review of the descriptive and treatment literature. Journal of Substance Abuse Treatment, 23, 231-237.

International Harm Reduction Association (2009). Global Overview: Drugs, HIV and Hepatitis C, 2009. Recuperado de http:/ / www.ihra.net/global-overview.

International Harm Reduction Association (2010). What is Harm Reduction: A position statement from the International Harm Reduction Association. IHRA Briefing, London United Kingdom, April 2010. Recuperado de http://www.ihra.net.

Jhanjee, S., Lal, T., Mondal, A. y Jain, K. (2011). Heroin-dependent family: a biopsychosocial context. Substance Abuse, 32, 157-158. doi:10.1080/08897077.2011.558450. 
Kokkevi, A. y Hartgers, C. (1995). EuropASI: European Adaptation of a Multidimensional Assessment Instrument for Drug and Alcohol Dependence. European Addiction Research, 1, 208-210.

Krug, A., Hildebrand, M. y Sun, N. (2015). "We don't need services. We have no problems": exploring the experiences of young people who inject drugs in accessing harm reduction services. Journal of the International AIDS Society, 18, S19442. doi:10.7448/IAS.18.2.19442.

Kwan, T. H., Wong, N. S. y Lee, S. S. (2015). Participation dynamics of a cohort of drug users in a low-threshold methadone treatment programme. Harm Reduction Journal, 12, 30. doi:10.1186/s12954-015-0072-z.

MacArthur, G. J., van Velzen, E., Palmateer, N., Kimber, J., Pharris, A., Hope, V., ... Hutchinson, S. J. (2014). Interventions to prevent HIV and Hepatitis $\mathrm{C}$ in people who inject drugs: a review of reviews to assess evidence of effectiveness. The International Journal on Drug Policy, 25, 34-52. doi:10.1016/j.drugpo.2013.07.001.

Marie-Claire, C., Crettol, S., Cagnard, N., Bloch, V., Mouly, S., Laplanche, J. L. ... Vorspan, F. (2016). Variability of response to methadone: genome-wide DNA methylation analysis in two independent cohorts. Epigenomics, 8,181-195. doi:10.2217/epi.15.110.

Martínez-Luna, N., Palma-Álvarez, R. F. y Roncero, C. (2016). Reducción de Daños. E: C. Roncero y M. Casas, ed., Patología Dual, Fundamentos Clínicos y Terapéuticos, 1st ed. Barcelona: Marge Medica Books, pp.253-266.

McNeil, R., Kerr, T., Pauly, B., Wood, E. y Small, W. (2016). Advancing patient-centered care for structurally vulnerable drug-using populations: a qualitative study of the perspectives of people who use drugs regarding the potential integration of harm reduction interventions into hospitals. Addiction, 111, 685-694. doi: 10.1111/ add.13214.

Miguel-Arias, D., Pereiro Gómez, C., Bermejo Barrera, A.M., López de Abajo Rodríguez, B. Y Sobrino Prieto, M. (2016). Mortality due to acute adverse drug reactions in Galicia: 1997-2011. Adicciones, 28, 80-89. doi:10.20882/ adicciones. 780 .

Observatorio Español de la Droga y las Toxicomanías. (2015). Informe 2015. Recuperado de http://www. pnsd.msssi.gob.es/profesionales/sistemasInformacion/ informesEstadisticas/pdf/INFORME_2015.pd.

Palmateer N., Kimber, J. Hickman, M., Hutchinson, S., Rhodes, T. y Goldberg, D. (2010). Evidence for the effectiveness of sterile injecting equipment provision in preventing hepatitis $\mathrm{C}$ and human immunodeficiency virus transmission among injecting drug users: a review of reviews. Addiction, 105, 844-859. doi:10.1111/j.13600443.2009.02888.x.

Parvaresen, N., Masoudi, A., Majidi-Tabrizi, S. y Mazhari, S. (2012). The Correlation between Methadone Dosage and Comorbid Psychiatric Disorders in Patients on Methadone Maintenance Treatment. Addiction and Health, 4, 1-8.

Peles E., Schreiber, S., Domany, Y. y Adelson, M. (2014). Impact of lifetime psychiatric diagnosis on long-term retention and survival of former opiate addicts in methadone maintenance treatment. The World Journal of Biological Psychiatry, 15, 629-635. doi:10.3109/15622975.2014.942359.

Pereiro, C., Pino, C., Flórez, G., Arrojo, M., Becoña, E. y COPSIAD Group. (2013). Psychiatric Comorbidity in Patients from the Addictive Disorders Assistance Units of Galicia: The COPSIAD Study. PloS One, 8, e66451. doi:10.1371/journal.pone.0066451.

Rhodes, T. y Hedrich, D. (2010). Harm Reduction: Evidence, Impact and Challenges. EMCDDA. Recuperado de http:/ /www.emcdda.europa.eu/publications/monographs/harm-reduction.

Roncero, C., Barral, C., Rodríguez-Cintas, L., Pérez-Pazos, J., Martinez-Luna, N., Casas, M., ... Grau-López, L. (2016). Psychiatric comorbidities in opioid-dependent patients undergoing a replacement therapy programme in Spain: The PROTEUS study. Psychiatry Research, 243, 174-181. doi:10.1016/j.psychres.2016.06.024.

Roncero, C., Daigre, C., Grau-López, L., Rodríguez-Cintas, L., Barral, C., Pérez-Pazos, J., ... Casas, M. (2013a). Cocaine-induced psychosis and impulsivity in cocaine-dependent patients. Journal of Addictive Diseases, 32, 263273. doi:10.1080/10550887.2013.824330.

Roncero, C., Fuste, G., Barral, C., Rodríguez-Cintas, L. Martínez-Luna, N. Eiroa-Orosa, F.J... Casas, M. on behalf of the PROTEUS study investigators. (2011). Therapeutic management and comorbidities in opiate-dependent patients undergoing a replacement therapy programme in Spain: the PROTEUS study. Heroin Addiction and Related Clinical Problems, 13, 5-16.

Roncero, C., Grau-López, L., Palma-Álvarez, R. F., Rodriguez-Cintas, L., Ros-Cucurull, E., Esojo, E. y Daigre, C. (2017). Higher severity of cocaine addiction is associated with tactile and somatic hallucination. European Psychiatry, 42, 63-69. doi:10.1016/j.eurpsy.2016.11.006.

Roncero, C., Martínez-Luna, N., Daigre, C., Grau-López, L., Gonzalvo, B., Pérez-Pazos, J. y Casas, M. (2013b). Psychotic symptoms of cocaine self-injectors in a harm reduction program. Substance Abuse, 34, 118-121. doi:10. 1080/08897077.2012.691446.

Salamina, G., Diecidue, R., Vigna-Taglianti, F., Jarre, P., Schifano, P., Bargagli, A. M., ... VEdeTTE Study Group. (2010). Effectiveness of therapies for heroin addiction in retaining patients in treatment: results from the VEdeTTE study. Substance Use E Misuse, 45, 2076-2092. doi:10.3109/10826081003791932.

San, L., Arranz, B., Arrojo, M., Becoña, E., Bernardo, M., Caballero, L., ... Zorrilla, I. (2016). Clinical guideline for the treatment of dual pathology in the adult population. Adicciones, 28, 3-5. doi:10.20882/adicciones.784. 
Seal, K. H., Kral, A. H., Gee, L., Moore, L. D., Bluthenthal, R. N., Lorvick, J. y Edlin, B. R. (2001). Predictors and prevention of nonfatal overdose among street-recruited injection heroin users in the San Francisco Bay Area, 1998-1999. American Journal of Public Health, 91, 18421846.

Teoh Bing Fei, J., Yee, A. y Habil, M. H. (2016). Psychiatric comorbidity among patients on methadone maintenance therapy and its influence on quality of life. American Journal of Addiction, 25, 49-55. doi:10.1111/ ajad.12317.

Torrens, M., Fonseca, F., Castillo, C. y Domingo-Salvany, A. (2013). Methadone maintenance treatment in Spain: the success of a harm reduction approach. Bulletin of the World Health Organization, 91, 136-141. doi:10.2471/ BLT.12.111054.

Wang, X., Zhang, T. y Ho, W. Z. (2011). Opioids and HIV/ HCV infection. Journal of Neuroimmune Pharmacology: The Official Journal of the Society on NeuroImmune Pharmacology, 6, 477-489. doi:10.1007/s11481-011-9296-1. 